BMJ Open Diabetes

Research \& Care

\section{Pioglitazone and cause-specific risk of mortality in patients with type 2 diabetes: extended analysis from a European multidatabase cohort study}

Helen Strongman, ${ }^{1}$ Solomon Christopher, ${ }^{2}$ Maila Majak, ${ }^{2}$ Rachael Williams, ${ }^{1}$ Shahram Bahmanyar, ${ }^{3}$ Marie Linder, ${ }^{3}$ Edith M Heintjes, ${ }^{4}$ Dimitri Bennett, ${ }^{5}$ Pasi Korhonen, ${ }^{2,6}$ Fabian Hoti $^{6}$
To cite: Strongman $\mathrm{H}$ Christopher S, Majak M, et al. Pioglitazone and cause-specific risk of mortality in patients with type 2 diabetes: extended analysis from a European multidatabase cohort study. BMJ Open Diab Res Care 2018;6:e000481. doi:10.1136/ bmjdrc-2017-000481

- Additional material is published online only. To view please visit the journal online (http://dx.doi.org/10.1136/ bmjdrc-2017-000481).

Received 4 October 2017 Revised 21 November 2017 Accepted 13 December 2017

Check for updates

${ }^{1}$ Clinical Practice Research Datalink (CPRD), London, UK ${ }^{2}$ EPID Research, Helsinki,

Finland

${ }^{3}$ Centre for

Pharmacoepidemiology, Karolinska Institutet, Stockholm, Sweden

${ }^{4}$ PHARMO Institute, Utrecht, The Netherlands

${ }^{5}$ Department of

Pharmacoepidemiology,

Takeda Pharmaceutical

Company Limited, Cambridge,

Massachusetts, USA

${ }^{6}$ EPID Research, Espoo, Finland

Correspondence to

Dr Fabian Hoti;

fabian.hoti@epidresearch.com

\section{ABSTRACT}

Objectives Describe and compare the risk of cardiovascular and non-cardiovascular mortality in patients whose antidiabetic therapy is modified to include pioglitazone compared with an alternative antidiabetic medication at the same stage of disease progression. Research design and methods This exploratory linked database cohort analysis used pooled health and mortality data from three European countries: Finland, Sweden and the UK. Propensity score together with exact matching was used to match 31133 patients with type 2 diabetes first prescribed pioglitazone from 2000 to 2011, to 31133 patients never prescribed pioglitazone. Exact matching variables were treatment stage, history of diabetes, diabetes complications and cardiovascular disease and year of cohort entry. Mean follow-up time was 2.60 (SD 2.00) and 2.69 (SD 2.31) years in the pioglitazone and non-pioglitazone-exposed groups, respectively. Crude cause-specific mortality rates were ascertained. Association with pioglitazone use was estimated using Cox proportional hazards models adjusted a priori for country, age, sex, the propensity score quintile and time-dependent variables representing use of antidiabetic drugs. Stepwise testing identified no additional confounders to include in adjusted models.

Results The crude mortality rate was lower in the pioglitazone-exposed group than the non-exposed group for both cardiovascular and non-cardiovascular mortality. Adjusted HRs comparing pioglitazone to alternative antidiabetic exposure were $0.58(95 \% \mathrm{Cl} 0.52$ to 0.63$)$ and $0.63(95 \% \mathrm{Cl} 0.58$ to 0.68$)$ for cardiovascular and noncardiovascular mortality, respectively. A protective effect associated with pioglitazone was also found for all specific cardiovascular causes.

Conclusions This analysis suggests that pioglitazone is associated with a decrease in both cardiovascular and non-cardiovascular mortality. Results should be interpreted with caution due to the potential for residual confounding in this exploratory analysis. Further studies, specifically designed to test the association between pioglitazone use and patient-focused outcomes, are suggested.

Study registration number European Network of Centres for Pharmacoepidemiology and Pharmacovigilance (ENCePP; EUPAS3626).

\section{Significance of this study}

What is already known about this subject?

- Evidence from a meta-analysis of seven randomized controlled trials indicates that pioglitazone exposure did not change the risk of death from any cause in patients with type 2 diabetes, pre-diabetes or insulin resistance. These trials were primary designed to study cardiovascular and composite outcomes.

- Previous secondary analyses of data from the original pan-European and other large retrospective cohort studies have demonstrated a statistically significant reduction in the risk of all-cause mortality with pioglitazone use.

- A range of exploratory studies have demonstrated that thiazolidinediones such as pioglitazone may have patient benefits beyond diabetes control and reduction of cardiovascular risk.

What are the new findings?

- This exploratory extension of the pan-European, observational, large cohort study suggests that exposure to pioglitazone compared with an alternative treatment decision at a similar stage of disease progression is associated with a statistically significant $42 \%$ and $37 \%$ reduction in cardiovascular and non-cardiovascular mortality, respectively.

How might these results change the focus of research or clinical practice?

- The suggestion of potentially broader benefits of pioglitazone supports its safe and effective use. Lack of agreement between randomized control trials and observational studies is likely to be due to reduced statistical power in the former and unresolved bias in the latter. Findings from this study can be used to inform study design for further research evaluating the association of pioglitazone use with specific long-term diagnostic and mortality outcomes. 


\section{INTRODUCTION}

Diabetes mellitus is one of the most common chronic diseases worldwide, and its global prevalence continues to rise due to the aging population, obesity, urbanization and changes associated with lifestyle such as physical inactivity. The increased risk of cardiovascular (CV) disease among patients with type 2 diabetes mellitus (T2DM) has been studied for many years and is well described in the literature. It is estimated that $\mathrm{CV}$ disease is responsible for some $70 \%$ of all mortality among patients with T2DM, ${ }^{1}$ and newly diagnosed adult diabetic patients have 5-10 years shorter life expectancy than the general population. ${ }^{23}$

We recently published results from a pan-European multidatabase study indicating no evidence of an association between pioglitazone use and bladder cancer risk $^{4}$ when compared with other antidiabetic drugs used in patients with T2DM. Further, in the same study, we found a statistically significant $33 \%$ reduction in all-cause mortality associated with pioglitazone use. ${ }^{5}$ This evidence of a reduction in risk of mortality supports other observational studies, ${ }^{67}$ though findings from a meta-analysis of seven trials demonstrate no significant difference in the risk of mortality with use of pioglitazone in patients with insulin resistance, pre-diabetes and T2DM compared with trial comparators. ${ }^{8}$

A recent review has summarized the findings of a large number of preclinical, clinical and observational studies that point to an overall beneficial effect of pioglitazone on CV disease in patients with T2DM and possible pleiotropic benefits. ${ }^{9}$ We report the findings of post-hoc analyses following from our original study findings of a reduced all-cause mortality associated with pioglitazone use, focusing on cause-specific mortality among patients with T2DM using data from Finland (FIN), Sweden (SWE) and the UK.

\section{METHODS}

\section{Study design and setting}

This exploratory linked database cohort analysis used three of the six non-overlapping datasets included in the bladder cancer and all-cause mortality analyses ${ }^{45}$ : FIN, SWE and the UK linked GP hospital dataset (UK GP-HOSP). Death registration data were not available for the remaining datasets. Data from the Clinical Practice Research Datalink (CPRD) were used in the UK.

\section{Data sources and outcome definition}

Drug exposure measures were generated from prescription data in the UK and dispensing data in FIN and SWE.

Cause-specific mortality was measured using the underlying cause of death field in linked national death registries in all countries. The following CV-related causes of death were classified using International Classification of Diseases, Tenth Revision (ICD-10) codes: myocardial infarction (MI), stroke, sudden death, congestive heart failure $(\mathrm{CHF})$ or cardiogenic shock, renovascular disease, peripheral vascular disease, arrhythmia and other CV (aortic aneurysm, other) (see online supplementary appendix table S1). All other causes of death were classified as non-CV. All causes of death are summarized by ICD-10 code chapter. Additional data from cancer registries, hospital discharge records, migration records and national diabetes registers were used, where available, to identify the study cohort and adjust for covariates. The study period start and end dates ranged respectively by dataset from 1 January 2000 to 1 July 2006 and from 31 December 2010 to 30 June 2011. The end date for the FIN dataset was brought forward by 6 months to December 2010 as cause of death data was not available after this point.

\section{Participants}

The study population comprised of patients with T2DM over the age of 40 whose therapy regimen was changed to include pioglitazone or any other antidiabetic medication during the study period. Potential cohort entry dates (CEDs) were identified as the date of the first ever pioglitazone prescription for pioglitazone-exposed patients and the date of switching to or add-on (initiation) of any other antidiabetic medication for the non-pioglitazone-exposed group. CEDs were excluded if there were $<12$ months' membership in the medication database, any indication of bladder cancer prior to CED or if the patients were concurrently exposed to another thiazolidinedione.

\section{Matching and covariates}

Guidelines recommend a stepwise approach to the prescribing of antidiabetic drugs that consider the drug substance's efficacy in controlling blood glucose and its safety profile. Clinicians therefore 'channel' pioglitazone to patients at a specified stage of disease progression and with other characteristics that modify their prognosis. As stated in the previous manuscript, the following approach was taken in an attempt to eliminate channeling bias. ${ }^{10}$

Propensity scores (PS) were estimated for each CED using a weighted logistic regression model with weights reciprocal to the number of CEDs per individual. The PS model included baseline binary and categorical variables that were likely to have a direct impact on treatment choice and could be measured in all datasets. These included antidiabetic treatment immediately prior to CED, whether the treatment change at CED was an add-on or switch in therapy, use of thiazolidinediones (TZDs) prior to CED, duration of treated diabetes, number of different antidiabetic medications used prior to CED, history of MI, stroke, heart failure and the following diabetic complications: retinopathy or maculopathy, severe lower limb complications, renal complications, ketoacidosis, and hyperosmolar or ketoacidotic coma, duration of prescription database membership and calendar year of CED.

Pioglitazone CEDs were 1:1 fixed ratio matched and 1:10 variable ratio matched to non-pioglitazone 
CEDs using the PS distance and the following exact matching variables: antidiabetic treatment immediately prior to CED, whether the treatment change at CED was an add-on or switch in therapy and use of TZDs prior to CED. Non-pioglitazone-exposed patients were removed from further matching when one of their CEDs was matched to a pioglitazone-exposed patient. The number of matched non-pioglitazone-exposed patients varied between patients and countries in the 1:10 matched cohort, resulting in an imbalance in characteristics. The main analysis was therefore based on the 1:1 fixed ratio matched cohort. Each patient was followed up from CED until date of death or censorship due to end of membership of the database, end of database coverage, start of other TZDs or 30 June 2011. Time-varying (TV) covariates were generated to improve the estimation of adjusted HRs. The following binary and categorical TV covariates were generated for inclusion or testing in the regression model: use of each alternative antidiabetic treatment class, cumulative duration of exposure to insulin and diagnosis of bladder cancer. Use of antidiabetic treatment group other than pioglitazone and comorbidity history were categorized as ever versus never used and ever versus never occurred up to any given time period. Age categories were fixed at baseline.

\section{Statistical analysis}

A pooled analysis was performed using the three datasets. Standardized differences were used to compare pioglitazone-exposed and never-exposed groups, with a score $<10$ indicating good balance. Crude cause-specific mortality rates were estimated with 95\% CI using Poisson regression. Cox proportional hazard models included as covariates age, gender, PS quintiles and TV use of antidiabetic medications. The proportional hazards assumption was examined by visually comparing the survival function for the pioglitazone-exposed and non-exposed groups. Further covariates, exact matching variables, PS variables and all variables that were included in the PS were included as confounders if their inclusion resulted in a minimum of $10 \%$ change in the HR of primary exposure (ever vs never exposed to pioglitazone) when introduced into the base model for the primary analysis for each cause-specific mortality outcome. Model selection was not repeated for stratified and sensitivity analyses. This differed from the all-cause mortality analyses in which exact matching and PS variables were automatically included in the adjusted model. History of chronic kidney disease/renalimpairment, diabetic complications and macrovascular disease were added to previous stratified analyses for duration of treated diabetes at CED, use of other TZDs prior to CED and history of renal complications at CED. Sensitivity analyses assessing the results with respect to exposure and the inclusion of baseline smoking, baseline body mass index (BMI) and TV HbAlc as covariates were supplemented with post-hoc analyses adjusting for a log transformed continuous age variable, removing patients with prior stroke, MI or CHF at CED and country-specific analyses.

\section{Further information}

Further details about the study methodology are published in the $B M f^{4}$ and in the European Network of Centres for Pharmacoepidemiology and Pharmacovigilance (ENCePP) E-register of studies (study title 'Pan-European Multi-Database Bladder Cancer Risk Characterisation Study').

\section{RESULTS}

The primary pooled matched cohort included 31133 patients in both the pioglitazone-exposed and the non-exposed groups. This followed removal of all patients in the NL and UK GP datasets, and 1900 and 1 matched pairs from the FIN and UK GP-HOSP cohorts respectively due to lack of availability of cause-specific mortality data for one or both members of the pair. The three-country cohorts are described in table 1 . The number of antidiabetic treatments prescribed at CED was similar in both cohorts. The most commonly prescribed treatment regimens in the never exposed to pioglitazone group were insulin alone or in combination $(37.5 \%)$, metformin and sulphonylureas (SU) in combination $(22.1 \%)$, or combinations of other antidiabetic drugs (30.7\%). Pioglitazone was prescribed in a variety of treatment regimens: most commonly in combination with metformin $(36.7 \%)$ or metformin and SU (25.5\%). Imbalance was observed in four of the variables used in the PS: MI or stroke at CED, CHF at CED, year of CED and duration of medication database membership prior to CED. The imbalance was due to reduced balance in key variables describing diabetic complications, history of macrovascular diseases and variables describing prior treatment in FIN (online supplementary appendix tables 2a-c).

In country-specific analyses, notable differences were observed in a number of covariates. History of TZD at cohort entry, a fixed matching variable, was less common in FIN $(17.3 \%)$ than in SWE $(38.1 \%)$ or the UK $(31.4 \%)$. Among the PS matching variables, diabetic complications were more common in the UK than in SWE and FIN. These differences were particularly pronounced for diabetic retinopathy or maculopathy (pioglitazone/non-pioglitazone): the UK 20.3\%/19.1\%; SWE 8.4\%/8.3\%; FIN 2.8\%/5.5\%; and diabetic renal complications: the UK $16.6 \% / 16.3 \%$; SWE $2.4 \% / 1.9 \%$; FIN $1.7 \% / 2.7 \%$. Full tables for each country are included in the online supplementary appendix tables S2a to S4c.

A total of 6176 deaths (1663 exposed and 4513 non-exposed) occurred during follow-up. This includes 2789 deaths due to CV-related causes (691 ever and 2098 never use of pioglitazone) and 3387 deaths due to non-CV causes (972 ever and 2415 never use of pioglitazone).

These events occurred over a mean 2.6 (SD 2.0) years of follow-up in the pioglitazone group and 2.7 (SD 2.3) in the non-pioglitazone-exposed group. The number of 
Table 1 Distribution of matching and non-matching covariates at cohort entry

\begin{tabular}{|c|c|c|c|}
\hline & $\begin{array}{l}\text { PIO exposed } \\
\mathrm{n}=31133 \mathrm{n}(\%)\end{array}$ & $\begin{array}{l}\text { Non-PIO-exposed } \\
\mathrm{n}=31133 \mathrm{n}(\%)\end{array}$ & $\begin{array}{l}\text { Standardized } \\
\text { difference }\end{array}$ \\
\hline \multicolumn{4}{|l|}{ Dataset } \\
\hline FIN & $15315(49.19)$ & $15315(49.19)$ & 0.00 \\
\hline SWE & $3712(11.92)$ & $3712(11.92)$ & 0.00 \\
\hline UK GP-HOSP & $12106(38.88)$ & $12106(38.88)$ & 0.00 \\
\hline \multicolumn{4}{|l|}{ Exact matching variables } \\
\hline \multicolumn{4}{|l|}{ Type of treatment change at CED } \\
\hline Add-on & $18774(60.30)$ & $18774(60.30)$ & 0.00 \\
\hline Switch & $12359(39.70)$ & $12359(39.70)$ & 0.00 \\
\hline \multicolumn{4}{|l|}{ History of TZD at CED } \\
\hline Never & 23261 (74.71) & 23261 (74.71) & 0.00 \\
\hline Ever & $7872(25.29)$ & $7872(25.29)$ & 0.00 \\
\hline \multicolumn{4}{|c|}{ Antidiabetic treatment immediately prior to $\mathrm{CED}^{*}$} \\
\hline Insulin (only or in combination) & $1456(4.68)$ & $1456(4.68)$ & 0.00 \\
\hline Metformin and SU & $7671(24.64)$ & $7671(24.64)$ & 0.00 \\
\hline Metformin only & $10036(32.24)$ & $10036(32.24)$ & 0.00 \\
\hline No treatment & $3636(11.68)$ & $3636(11.68)$ & 0.00 \\
\hline Other drugs or combination & $5405(17.36)$ & $5405(17.36)$ & 0.00 \\
\hline SU only & $2929(9.41)$ & $2929(9.41)$ & 0.00 \\
\hline \multicolumn{4}{|l|}{ Propensity score variables } \\
\hline \multicolumn{4}{|c|}{ Duration of treated diabetes at CED (years) } \\
\hline$<1$ & $3833(12.31)$ & $3870(12.43)$ & 0.36 \\
\hline 1 to $<2$ & $3923(12.60)$ & $3571(11.47)$ & 3.48 \\
\hline 2 to $<4$ & $6333(20.34)$ & $5957(19.13)$ & 3.03 \\
\hline 4 to $<6$ & $6379(20.49)$ & $7017(22.54)$ & 4.99 \\
\hline$\geq 6$ & $10665(34.26)$ & $10718(34.43)$ & 0.36 \\
\hline Range (min, max) & $(0.00,34.18)$ & $(0.00,24.79)$ & \\
\hline Mean $( \pm S D)$ & $4.77(3.61)$ & $4.86(3.62)$ & \\
\hline Median (Q1, Q3) & $4.24(1.80,7.05)$ & $4.43(1.94,7.02)$ & \\
\hline \multicolumn{4}{|l|}{ History of diabetic complications at CED } \\
\hline Diabetic retinopathy or maculopathy & $3189(10.24)$ & $3460(11.11)$ & 2.82 \\
\hline Lower limb severe complications & $750(2.41)$ & $958(3.08)$ & 4.09 \\
\hline Diabetic renal complications & $2351(7.55)$ & $2452(7.88)$ & 1.22 \\
\hline Ketoacidosis & $74(0.24)$ & $116(0.37)$ & 2.45 \\
\hline Hyperosmolar/ketoacidotic coma & $661(2.12)$ & $1097(3.52)$ & 8.46 \\
\hline \multicolumn{4}{|l|}{ Other comorbidities at CED } \\
\hline Myocardial infarction or stroke & $2687(8.63)$ & $3853(12.38)$ & 12.24 \\
\hline Congestive heart failure & $1069(3.43)$ & $2213(7.11)$ & 16.50 \\
\hline \multicolumn{4}{|l|}{ Year at CED } \\
\hline 2000-2003 & $1672(5.37)$ & $4103(13.18)$ & 27.17 \\
\hline 2004-2007 & $11214(36.02)$ & $10573(33.96)$ & 4.32 \\
\hline 2008-2011 & $18247(58.61)$ & $16457(52.86)$ & 11.59 \\
\hline \multicolumn{4}{|c|}{$\begin{array}{l}\text { Duration of medication database membership before } \\
\text { CED (years) }\end{array}$} \\
\hline $1-2$ & $2538(8.15)$ & 2528 (8.12) & 0.12 \\
\hline
\end{tabular}


Table 1 Continued

\begin{tabular}{|c|c|c|c|}
\hline & $\begin{array}{l}\text { PIO exposed } \\
\mathrm{n}=31133 \mathrm{n}(\%)\end{array}$ & $\begin{array}{l}\text { Non-PIO-exposed } \\
\mathrm{n}=31133 \mathrm{n}(\%)\end{array}$ & $\begin{array}{l}\text { Standardized } \\
\text { difference }\end{array}$ \\
\hline $3-4$ & $2353(7.56)$ & $3184(10.23)$ & 9.39 \\
\hline $5-6$ & $3930(12.62)$ & $5912(18.99)$ & 17.52 \\
\hline $7+$ & $22312(71.67)$ & $19509(62.66)$ & 19.26 \\
\hline Range (min, max) & $(1.00,22.00)$ & $(1.00,22.00)$ & \\
\hline Mean $( \pm \mathrm{SD})$ & $8.86(4.13)$ & $8.37(4.13)$ & \\
\hline Median (Q1, Q3) & $9.00(6.00,11.00)$ & $8.00(5.00,11.00)$ & \\
\hline \multicolumn{4}{|c|}{$\begin{array}{l}\text { Different antidiabetic drug classes ever prior to } \\
\text { CED (n) }\end{array}$} \\
\hline 0 & $1097(3.52)$ & $1061(3.41)$ & 0.63 \\
\hline 1 & $8928(28.68)$ & $7833(25.16)$ & 7.94 \\
\hline 2 & $13754(44.18)$ & $12832(41.22)$ & 5.99 \\
\hline 3 & $5833(18.74)$ & $7204(23.14)$ & 10.84 \\
\hline$>3$ & $1521(4.89)$ & $2203(7.08)$ & 9.25 \\
\hline \multicolumn{4}{|c|}{ Non-matching study variables (selected) } \\
\hline \multicolumn{4}{|l|}{ Age at CED (years) } \\
\hline $40-59$ & $12073(38.78)$ & $9703(31.17)$ & 16.01 \\
\hline $60-69$ & $10355(33.26)$ & $9506(30.53)$ & 5.85 \\
\hline$\geq 70$ & $8705(27.96)$ & $11924(38.30)$ & 22.10 \\
\hline Range (min, max) & $(40.00,98.00)$ & $(40.00,101.00)$ & \\
\hline Mean $( \pm \mathrm{SD})$ & $63.17(10.68)$ & $65.89(11.53)$ & \\
\hline Median (Q1, Q3) & $63.00(56.00,71.00)$ & $66.00(58.00,75.00)$ & \\
\hline \multicolumn{4}{|l|}{ Sex } \\
\hline Male & $17989(57.78)$ & $17127(55.01)$ & 5.59 \\
\hline Female & $13144(42.22)$ & $14006(44.99)$ & 5.59 \\
\hline \multicolumn{4}{|c|}{$\begin{array}{l}\text { Different antidiabetic drug classes being used at } \\
\text { CED† }(n)\end{array}$} \\
\hline 1 & $3242(10.41)$ & $4727(15.18)$ & 14.31 \\
\hline 2 & $15776(50.67)$ & $16482(52.94)$ & 4.54 \\
\hline 3 & $10862(34.89)$ & $9494(30.49)$ & 9.38 \\
\hline$>3$ & $1253(4.02)$ & $430(1.38)$ & 16.36 \\
\hline \multicolumn{4}{|l|}{ Antidiabetic treatment at CED $\dagger$} \\
\hline Insulin (only or in combination) & $1462(4.70)$ & $11662(37.46)$ & 87.71 \\
\hline Metformin and SU & $7925(25.46)$ & $6872(22.07)$ & 7.95 \\
\hline Metformin only & 11439 (36.74) & $2007(6.45)$ & 79.19 \\
\hline No treatment & $3242(10.41)$ & $0(0.00)$ & 48.22 \\
\hline Other drugs or combination & $3830(12.30)$ & $9571(30.74)$ & 46.04 \\
\hline SU only & $3235(10.39)$ & $1021(3.28)$ & 28.46 \\
\hline \multicolumn{4}{|l|}{ Bladder comorbidities at CED } \\
\hline Urinary incontinence & $1941(6.23)$ & $2336(7.50)$ & 5.02 \\
\hline Urinary tract infection & $2458(7.90)$ & $2818(9.05)$ & 4.15 \\
\hline Pyelonephritis & $458(1.47)$ & $604(1.94)$ & 3.62 \\
\hline Urolithiasis & $809(2.60)$ & $723(2.32)$ & 1.78 \\
\hline Hematuria & 1118 (3.59) & $1192(3.83)$ & 1.26 \\
\hline Urinary retention & $369(1.19)$ & $530(1.70)$ & 4.34 \\
\hline Neurogenic bladder & $40(0.13)$ & $50(0.16)$ & 0.85 \\
\hline
\end{tabular}


Table 1 Continued

\begin{tabular}{|c|c|c|c|}
\hline & $\begin{array}{l}\text { PIO exposed } \\
n=31133 n(\%)\end{array}$ & $\begin{array}{l}\text { Non-PIO-exposed } \\
n=31133 \mathrm{n}(\%)\end{array}$ & $\begin{array}{l}\text { Standardized } \\
\text { difference }\end{array}$ \\
\hline Catheterization & $321(1.03)$ & $450(1.45)$ & 3.75 \\
\hline \multicolumn{4}{|l|}{ Other comorbidities at CED } \\
\hline Other urinary tract cancer (excluding BC) & $96(0.31)$ & $105(0: 34)$ & 0.51 \\
\hline Other cancer (excluding urinary tract) & $3415(10.97)$ & $4078(13.10)$ & 6.55 \\
\hline COPD & $2729(8.77)$ & $2959(9.50)$ & 2.56 \\
\hline \multicolumn{4}{|l|}{ Use of other medications prior to CED } \\
\hline Statins & $14933(47.97)$ & $13832(44.43)$ & 7.10 \\
\hline ARB & $9066(29.12)$ & $7856(25.23)$ & 8.74 \\
\hline ACE inhibitors & $17762(57.05)$ & 18004 (57.83) & 1.57 \\
\hline $\mathrm{BPH}$ & 4108 (13.20) & 4272 (13.72) & 1.54 \\
\hline
\end{tabular}

*Treatments initiated at CED are not included.

†Treatments initiated at CED are included. For the PIO-exposed group, the listed treatments are in addition to PIO.

$\mathrm{ARB}$, angiotensin receptor blockers; BC, bladder cancer; $\mathrm{BPH}$, benign prostatic hypertrophy; CED, cohort entry date; COPD, chronic obstructive pulmonary disease; FIN, Finland; PIO, pioglitazone; SU, sulphonylureas; SWE, Sweden; TZD, thiazolidinedione; UK GP-HOSP, UK linked dataset.

mortality events by ICD-10 chapter is provided in table 2 . The most common causes of death were circulatory system-related deaths $(45.9 \%)$ and neoplasms $(26.5 \%)$.

Table 2 Number and percentage of deaths by International Classification of Diseases, Tenth Revision (ICD-10) chapter

\begin{tabular}{|c|c|c|}
\hline Cause of death & ICD-10 code & N (\%) \\
\hline Total & & $6176(100.00 \%)$ \\
\hline Circulatory system & $100-199$ & $2834(45.89 \%)$ \\
\hline Neoplasm & C00 - D48 & 1635 (26.47\%) \\
\hline Respiratory & J00-J99 & $359(5.81 \%)$ \\
\hline $\begin{array}{l}\text { Endocrine, nutritional and } \\
\text { metabolic }\end{array}$ & E00-E90 & $332(5.38 \%)$ \\
\hline Digestive & K00-K93 & $248(4.02 \%)$ \\
\hline External cause & V01-Y98 & $228(3.69 \%)$ \\
\hline Nervous system & G00-G99 & $179(2.90 \%)$ \\
\hline Mental and behavioral & F00-F99 & $144(2.33 \%)$ \\
\hline Genitourinary & N00-N99 & $85(1.38 \%)$ \\
\hline Infectious disease & A00-B99 & $55(0.89 \%)$ \\
\hline Not classified elsewhere & R00-R99 & $28(0.45 \%)$ \\
\hline $\begin{array}{l}\text { Muscoskeletal and } \\
\text { connective tissue }\end{array}$ & M00-M99 & $24(0.39 \%)$ \\
\hline $\begin{array}{l}\text { Skin and subcutaneous } \\
\text { tissue }\end{array}$ & L00-L99 & $14(0.23 \%)$ \\
\hline $\begin{array}{l}\text { Blood, blood-forming } \\
\text { organs and immune }\end{array}$ & D50-D89 & $7(0.11 \%)$ \\
\hline $\begin{array}{l}\text { Congenital malformations, } \\
\text { deformations and } \\
\text { chromosomal } \\
\text { abnormalities }\end{array}$ & Q00-Q99 & $3(0.05 \%)$ \\
\hline Eye and ear & $\mathrm{H} 00-\mathrm{H} 95$ & $1(0.02 \%)$ \\
\hline
\end{tabular}

\section{CV versus non-CV mortality}

The number of CV and non-CV events and their corresponding crude mortality rates are provided in table 3 . The rate was lower in the pioglitazone-exposed group than the non-exposed group for both $\mathrm{CV}$ and non-CV mortality. The Kaplan-Meier curves for CV and non-CV mortality are consistent with the proportional hazards assumption (figure 1A,B).

The adjusted HR for ever exposed compared with never exposed to pioglitazone was 0.60 (95\% CI 0.57 to 0.64 ) for all-cause mortality, 0.58 (95\% CI 0.52 to 0.63$)$ for CV mortality and 0.63 (95\% CI 0.58 to 0.68 ) for non-CV mortality (table 4). No additional variables met the confounding inclusion criteria so the base model was considered the final model. All three HR estimates were statistically significant and indicated a decrease in the risk of mortality associated with pioglitazone exposure. The protective effect was strongest in the longest duration of exposure and highest cumulative dose categories (table 4). Discontinuation of pioglitazone use within the last year was associated with an increase in the mortality risk.

Significant interactions were observed in stratified analyses between pioglitazone exposure and the stratification variables (table 5). The reduced risk of all-cause, $\mathrm{CV}$ and non-CV mortality associated with pioglitazone use was stronger in patients with no history of diabetic complications, no history of macrovascular diseases and no history of chronic kidney disease or renal impairment. Duration of treated diabetes at CED, classified as $<4$ years and $\geq 4$ years, was not found to interact with the observed associations. The risk reduction for non-CV mortality was lower in patients with no history of other TZD use. There was a directional but not significant difference in the risk reduction for $\mathrm{CV}$ mortality in the ever versus never received TZD at cohort entry groups. 
Table 3 Number of cardiovascular and non-cardiovascular deaths and their corresponding crude mortality rates

PIO-exposed Non-PIO-exposed

\begin{tabular}{|c|c|c|c|c|}
\hline Patients (n) & 31333 & & 31333 & \\
\hline Patient years (mean, SD) & $2.6(2.0)$ & & $2.7(2.3)$ & \\
\hline \multirow[t]{2}{*}{ Patient years (median, IQR) } & $2.3(0.9-3.9)$ & & $2.1(0.8-4.1)$ & \\
\hline & Deaths (n) & $\begin{array}{l}\text { Mortality rate per } 10000 \\
\text { patient-years }(95 \% \mathrm{Cl})\end{array}$ & Deaths (n) & $\begin{array}{l}\text { Mortality rate per } 10000 \\
\text { patient-years }(95 \% \mathrm{Cl})\end{array}$ \\
\hline Cardiovascular & 691 & 85.42 (79.28 to 92.03$)$ & 2098 & 250.51 (240.02 to 261.46$)$ \\
\hline Non-cardiovascular & 972 & 120.15 (112.83 to 127.95$)$ & 2415 & 288.36 (277.09 to 300.1$)$ \\
\hline All-cause & 1663 & 205.57 (195.92 to 215.69 ) & 4513 & 538.87 (523.38 to 554.82$)$ \\
\hline
\end{tabular}

$\mathrm{PIO}$, pioglitazone.

A statistically significant reduction in mortality risk was observed in all stratified analyses (table 5) with the exception of use of TZDs prior to cohort entry for non-CV mortality (HR $0.86,95 \%$ CI $0.69,1.08$ ) and sensitivity analyses (online supplementary appendix tables S5a and S5b). These included country-specific analyses (table 6). For CV-specific mortality, relative risk reduction ranged from $7 \%$ in the UK GP-HOSP dataset to $65 \%$ in SWE. For non-CV mortality, the range was from $17 \%$ in SWE to $51 \%$ in FIN. In FIN and UK GP-HOSP, the HR for CV mortality is similar to the HR for non-CV mortality. There was a considerable difference between the HRs in SWE where the protective effect was strongest for CV mortality.

\section{CV mortality subanalyses}

The most common CV cause of death in each group was MI followed by stroke and heart failure. Together these accounted for $88 \%$ of $\mathrm{CV}$ deaths in both the pioglitazone-exposed and non-exposed group. The crude mortality rate was lower in the pioglitazone-exposed group than the non-exposed group for each $\mathrm{CV}$ cause (table 7).

The adjusted HRs for MI, stroke and heart failure were $0.61(95 \%$ CI 0.55 to 0.69$), 0.48$ (95\% CI 0.38 to 0.62$)$ and 0.60 (95\% CI 0.42 to 0.86$)$, respectively. A decreased risk of mortality was observed for all CV causes with statistical significance demonstrated in all except renovascular disease and peripheral vascular disease (figure 2). There were no clear trends with increasing duration of pioglitazone use or with increasing cumulative dose (online supplementary appendix table S6).

Preplanned stratified analyses were restricted to MI and stroke due to low numbers of deaths in other categories (online supplementary appendix table S7a and S7b). The reduced risk of MI-related mortality associated with pioglitazone use was stronger in patients with no history of macrovascular diseases and for patients with no history of diabetic complications. Stratified analyses for stroke-related mortality were consistent with the main findings. Sensitivity analyses completed for MI, stroke and heart failure demonstrated a directional or statistically significant protective effect of pioglitazone in all analyses with the exception of MI in the UK (HR 1.05,
95\% CI 0.86 to 1.28 ) (online supplementary appendix tables S8a and S8b).

\section{DISCUSSION}

In this post-hoc analysis using a large three-country European cohort of patients with T2DM, we found a statistically significant $42 \%$ and $37 \%$ reduction in risk of CV and non-CV mortality, respectively, in patients whose antidiabetic therapy was modified to include pioglitazone compared with an alternative antidiabetic medication at a similar stage of disease progression. Comparators included a full range of antidiabetic treatment regimens from metformin alone $(6 \%)$ to insulin used alone or in combination $(37 \%)$. We also observed a statistically significant $39 \%, 52 \%$ and $40 \%$ reduction for MI, stroke and heart failure cause-specific mortality.

There was no evidence of a consistent trend in mortality risk ( $\mathrm{CV}$ or non-CV) with increasing duration of pioglitazone use or with increasing cumulative dose of pioglitazone. Discontinuation of exposure to pioglitazone within the last year was associated with an increase in the mortality risk. This may be due to switch to insulin therapy for end-stage patients or undocumented pioglitazone treatment during hospitalization that could be missing in drug prescription/dispensing registers.

Strengths of the primary study ${ }^{4}$ include the large number of patients included in the study, long follow-up period (up to 10 years) and extensive measures to reduce or investigate potential biases. These include a PS matched cohort design to minimize channeling bias related to factors that were hypothesized to influence prescribing decisions and be associated with the bladder cancer outcome, which was the outcome of interest in the main pan-European study. This resulted in the pioglitazone-exposed groups in the matched cohort for the bladder cancer analysis being balanced with regards to the distribution of measured confounders included as exact matching variables or in the PS. Marginal exceptions included CHF at baseline, early cohort entry (2000-2003) and higher duration of medication database membership before CED (5-6 and 7+years), where the standardized differences marginally exceeded 10 . The design included 

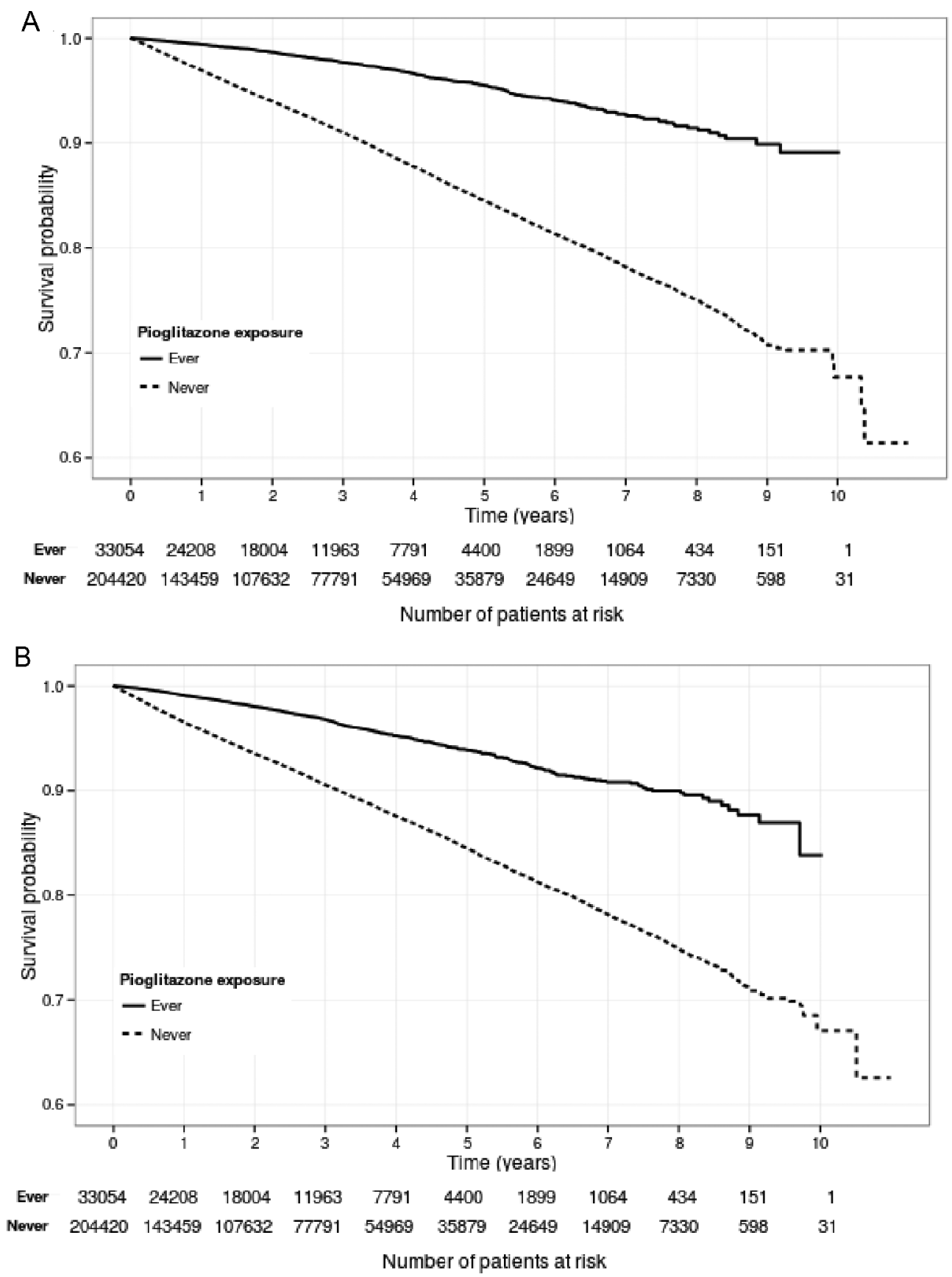

Figure 1 Kaplan-Meier plots of association of exposure to pioglitazone with (A) cardiovascular (CV) mortality and (B) non-CV mortality in patients with type 2 diabetes. Ever, exposed to pioglitazone; Never, never exposed to pioglitazone.

further adjustment for matching variables and additional baseline and TV confounders in the main regression model. We also ran numerous stratifications to identify potential effect modification and sensitivity analyses to evaluate robustness of study results. These included an assessment of the impact of BMI, smoking status and TV HbAlc as covariates in countries where these variables were available. Remaining limitations were the potential for residual confounding due to unmeasured covariates such as general health and socioeconomic status ${ }^{11}$; and left truncation of prescribing data, particularly in SWE where data collection started in July $2005 .{ }^{4}$ This left truncation may have led to misclassification of incident pioglitazone exposure despite the requirement for at least 1 year of baseline data prior to index.

These strengths and limitations broadly apply to this extended analysis as well. However, the covariates and likely mechanisms through which they influence the association between pioglitazone exposure and outcomes differ for bladder cancer and specific causes of mortality. For example, urinary tract or other cancers diagnosed either prior to baseline or developing during follow-up were not found to be significant confounders in the primary analyses for each cause-specific mortality outcome and therefore not included in the final models. In a study designed specifically to test the association 
Table 4 Adjusted ${ }^{*}$ HRs for cardiovascular (CV) mortality, non-CV mortality and all-cause mortality

\begin{tabular}{|c|c|c|c|}
\hline Exposure definitions & $\begin{array}{l}\text { CV mortality } \\
\text { HR (95\% CI) }\end{array}$ & $\begin{array}{l}\text { Non-CV mortality } \\
\text { HR }(95 \% \mathrm{Cl})\end{array}$ & $\begin{array}{l}\text { All-cause mortality } \\
\text { HR }(95 \% \mathrm{Cl})\end{array}$ \\
\hline \multicolumn{4}{|l|}{ Pioglitazone exposure } \\
\hline Never & Reference & Reference & Reference \\
\hline Ever & $0.58(0.52$ to 0.63$)$ & 0.63 (0.58 to 0.68$)$ & 0.60 (0.57 to 0.64$)$ \\
\hline \multicolumn{4}{|c|}{ Pioglitazone current exposure } \\
\hline No & Reference & Reference & Reference \\
\hline Yes & 0.41 (0.36 to 0.47$)$ & $0.30(0.26$ to 0.34$)$ & 0.34 (0.31 to 0.38$)$ \\
\hline \multicolumn{4}{|c|}{ Duration of pioglitazone exposure (months) } \\
\hline Never & Reference & Reference & Reference \\
\hline$<12$ & 0.56 (0.50 to 0.63$)$ & 0.65 (0.58 to 0.71$)$ & 0.61 (0.56 to 0.66$)$ \\
\hline $12-24$ & $0.64(0.54$ to 0.76$)$ & 0.61 (0.53 to 0.71$)$ & $0.62(0.56$ to 0.70$)$ \\
\hline $24-48$ & $0.59(0.49$ to 0.71$)$ & 0.66 (0.57 to 0.78$)$ & $0.63(0.56$ to 0.71$)$ \\
\hline$>48$ & $0.44(0.32$ to 0.61$)$ & $0.45(0.34$ to 0.60$)$ & $0.44(0.36$ to 0.55$)$ \\
\hline \multicolumn{4}{|c|}{ Cumulative pioglitazone dose (mg) } \\
\hline Never & Reference & Reference & Reference \\
\hline $1-10500$ & 0.57 (0.51 to 0.64$)$ & 0.64 (0.58 to 0.71$)$ & 0.61 (0.57 to 0.66$)$ \\
\hline $10501-28000$ & 0.63 (0.54 to 0.73$)$ & 0.66 (0.58 to 0.76$)$ & 0.64 (0.58 to 0.71$)$ \\
\hline $28001-40000$ & $0.54(0.40$ to 0.72$)$ & $0.62(0.48$ to 0.79$)$ & $0.58(0.48$ to 0.70$)$ \\
\hline$>40000$ & $0.48(0.36$ to 0.66$)$ & $0.43(0.32$ to 0.57$)$ & 0.45 (0.37 to 0.55$)$ \\
\hline \multicolumn{4}{|c|}{ Time since last exposure (years) } \\
\hline Never & Reference & Reference & Reference \\
\hline Current use & $0.38(0.33$ to 0.44$)$ & $0.30(0.27$ to 0.35$)$ & $0.34(0.31$ to 0.37$)$ \\
\hline$<1$ & 0.95 (0.81 to 1.10$)$ & $1.34(1.20$ to 1.50$)$ & $1.17(1.07$ to 1.28$)$ \\
\hline $1-2$ & 0.61 (0.49 to 0.75$)$ & 0.75 (0.62 to 0.89$)$ & $0.68(0.59$ to 0.78$)$ \\
\hline $2-4$ & 0.66 (0.53 to 0.80$)$ & 0.74 (0.62 to 0.89$)$ & 0.70 (0.61 to 0.80$)$ \\
\hline$>4$ & 0.77 (0.58 to 1.04$)$ & 0.71 (0.54 to 0.93$)$ & 0.73 (0.60 to 0.90$)$ \\
\hline
\end{tabular}

${ }^{*}$ Adjusted for dataset/country, propensity score quintiles, age at cohort entry dates, sex and ever exposed to metformin, sulphonylureas, insulin and other non-insulin diabetic treatments.

of pioglitazone use with all cancers, patients diagnosed with cancer prior to baseline would likely be removed. There is also a potential risk that residual confounders such as general health and severity of pre-existing conditions have a stronger impact on the association between pioglitazone use and cause-specific mortality and may partially contribute to the observed association. Conversely, there is an increased risk that covariates included in the PS model were not associated with the mortality outcomes. In order to minimize the effect of this, exact matching and individual PS variables were not automatically included in the adjusted models in the analyses reported here. They were tested as confounders using a stepwise methodology but did not meet the threshold for inclusion in the final model. This change in approach decreased the effect size for all-cause mortality from $25 \%$ reported in the all-cause mortality manuscript ${ }^{5}$ to $14 \%$ in the UK-HOSP dataset. In FIN, we observed an increase in effect from $46 \%$ to $52 \%$ which could be due to one or both of this change in methodology or the loss of matched pairs with CEDs in 2011 and resulting loss of covariate balance in multiple PS covariates, notably MI or stroke at baseline, CHF at baseline, year of CED and duration of medication database membership prior to CED. There was no change in SWE.

$\mathrm{CV}$ and non-CV mortality risk reductions were observed in all countries. In SWE, this reduction was statistically significant for $\mathrm{CV}$ mortality risk (HR $0.35,95 \%$ CI 0.22 to 0.56 ) but smaller and directional for non-CV mortality risk (HR $0.83,95 \%$ CI 0.62 to 1.10 ). Similar differences between $\mathrm{CV}$ and non-CV mortality risk were not observed in FIN and the UK, although both effect sizes were larger in FIN and the risk reduction for CV mortality risk was not significant in the UK.

Stratified analyses demonstrated statistically significant effect modification by history of diabetic complications, chronic kidney disease and macrovascular disease for both CV and non-CV mortality, with a weaker risk reduction associated with pioglitazone use in patients with a history of these conditions. Diabetic complications and renal disease were more common in the UK than in SWE and FIN. The reduced risk 
Table 5 Adjusted HRs for all-cause, cardiovascular (CV) and non-CV mortality in each of the stratified analyses

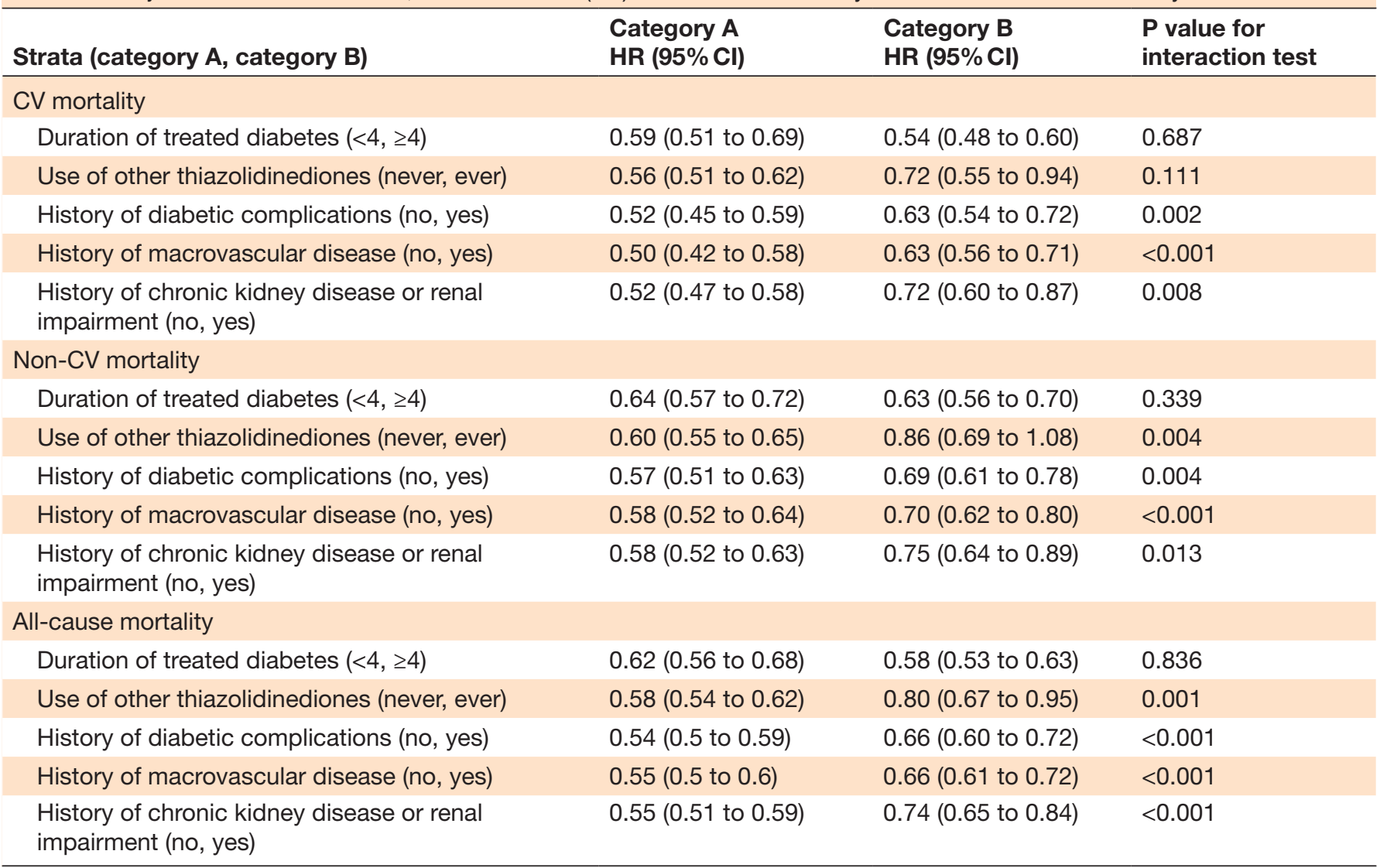

of both CV and non-CV mortality was weaker in the UK sensitivity analysis. Given the use of primary care data in the UK in addition to the secondary care data sources used in all three countries, these differences may in part reflect differences in data recording. This may lead to variation in PS modeling and matching with the UK model better able to minimize channeling bias. Conversely misclassification of exposure may be higher in the UK where analyses rely on prescribing data than in FIN and SWE where dispensing data were used. Assuming that misclassification was non-differential, this would attenuate the observed protective effect in the UK. Country differences may also reflect true differences in diabetic complications and renal disease attenuating the beneficial effect of pioglitazone or differences in other characteristics and care received by patients prescribed pioglitazone in the three countries. Cause of death registers in all three countries use WHO ICD-10 to code underlying cause of death and follow internationally agreed rules. ${ }^{12-15}$ Differential coding of cause of death between countries and between pioglitazone-exposed and unexposed groups is therefore unlikely.

Stratified analyses for history of TZD at cohort entry showed a stronger protective effect with pioglitazone use in patients with no history of TZD use for non-CV mortality where the effect was statistically significant and CV mortality where the effect was directional. For CV mortality, this risk difference may be explained by the propensity of rosiglitazone to increase $\mathrm{CV}$ risk which led to its withdrawal from the UK market in 2010. ${ }^{11}$ Interestingly, history of TZD use at cohort entry was less common in FIN than in SWE and the UK and the risk reduction for

Table 6 Adjusted HRs for all-cause, cardiovascular (CV) and non-CV mortality in each dataset

\begin{tabular}{llll} 
Sensitivity analysis & $\begin{array}{l}\text { CV mortality } \\
\text { HR }(\mathbf{9 5 \%} \mathbf{C l})\end{array}$ & $\begin{array}{l}\text { Non-CV mortality } \\
\text { HR (95\% Cl) }\end{array}$ & $\begin{array}{l}\text { All-cause } \\
\text { HR (95\% Cl) }\end{array}$ \\
\hline FIN & $0.46(0.41$ to 0.53$)$ & $0.49(0.44$ to 0.55$)$ & $0.48(0.44$ to 0.52$)$ \\
SWE & $0.35(0.22$ to 0.56$)$ & $0.83(0.62$ to 1.10$)$ & $0.63(0.49$ to 0.80$)$ \\
UK GP-HOSP & $0.93(0.79$ to 1.09$)$ & $0.82(0.72$ to 0.94$)$ & $0.86(0.78$ to 0.95$)$ \\
Pooled data & $0.58(0.52$ to 0.63$)$ & $0.63(0.58$ to 0.68$)$ & $0.60(0.57$ to 0.64$)$ \\
\hline
\end{tabular}

FIN, Finland; SWE, Sweden; UK GP-HOSP, UK linked dataset. 
Table 7 Number of cardiovascular cause-specific deaths and their corresponding crude mortality rates per 10000 personyears

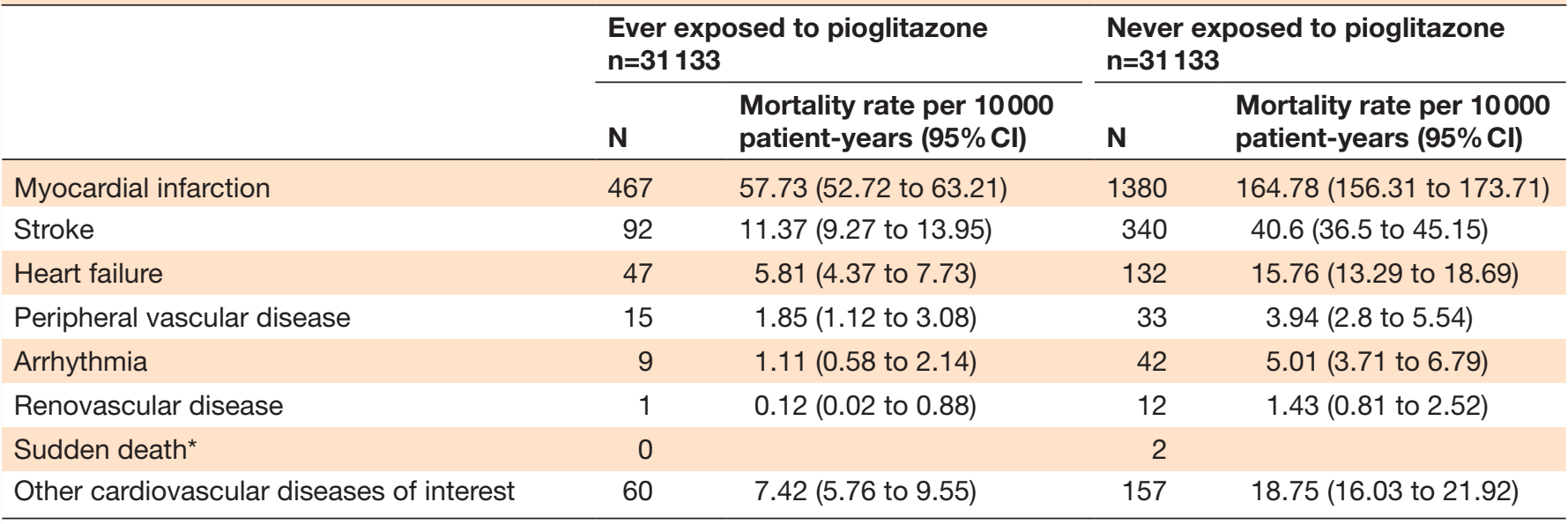

*Mortality not calculated due to lack of statistical convergence of the model.

non-CV mortality was greater in the FIN sensitivity analysis.

Liao et al recently published a meta-analysis of nine randomized controlled trials evaluating the effect of pioglitazone on $\mathrm{CV}$ and safety outcomes. This meta-analysis demonstrated a significantly reduced risk of major CV events (composite of non-fatal MI, non-fatal stroke and CV death) in patients with diabetes (relative risk (RR) $0.83,95 \%$ CI 0.72 to 0.97 ) and pre-diabetes or insulin resistance (RR $0.77,95 \%$ CI 0.64 to 0.93$){ }^{8}$ This is in line with our finding of a protective effect for pioglitazone on CV mortality compared with other antidiabetic treatments prescribed at similar stage of disease progression. The meta-analysis also demonstrated an increased risk of CHF with pioglitazone (RR 1.32; CI 1.14 to 1.54 ). This contradicts our findings of a protective effect for CHF mortality (HR $0.60,95 \%$ CI 0.42 to 0.86 ), perhaps reflecting adherence to warnings about the safety of pioglitazone prescribing in patients with heart failure when used in the real-world setting, following publication of the PROspective pioglitAzone Clinical Trial In macroVascular Events (PROactive) randomized control trial. ${ }^{9}$

There is little evidence from randomized controlled trials and observational studies examining the association of pioglitazone with non-CV patient outcomes and mortality with which to compare findings from this study. However, no statistically significant difference was observed in the rate of all-cause mortality in Liao et $a l$ 's meta-analysis (seven trials; RR $0.93,95 \%$ CI 0.80 to 1.09). ${ }^{8}$ The vast majority $(90 \%)$ of the 11319 subjects in the meta-analysis were enrolled in two large trials: the PROactive ${ }^{1617}$ trial in patients with type 2 diabetes at high risk of $\mathrm{CV}$ disease and the Insulin Resistance Intervention after Stroke (IRIS) trial in pre-diabetic patients with a history of ischemic stroke or transient ischemic attack. The result from the meta-analysis contradicts the findings from the current analysis and other large observational studies with long follow-up periods that have demonstrated significant reductions in all-cause mortality risk with pioglitazone use over

Causes of death

Adjusted HR (95\% Cl)

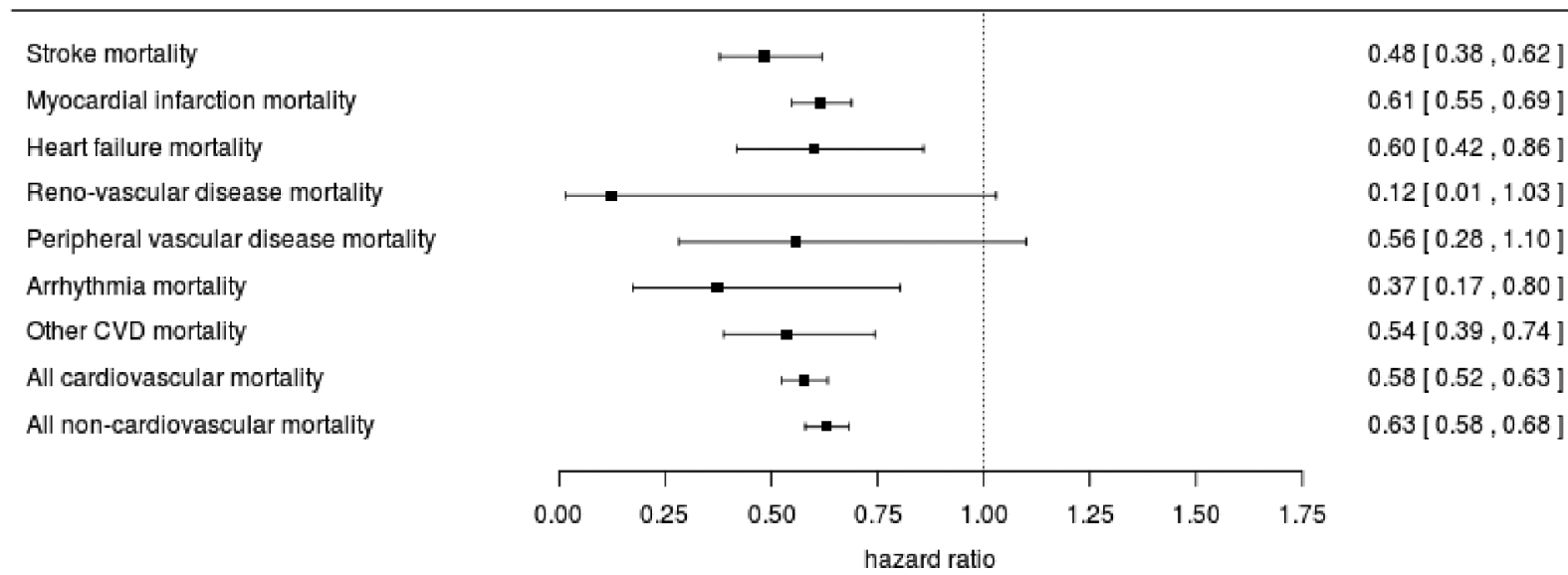

Figure 2 Adjusted HRs with 95\% Cl for the association between pioglitazone use and cause-specific mortality. CVD, cardiovascular disease. 
longer follow-up periods. ${ }^{5-7}$ This lack of agreement is likely to be due to a combination of reduced statistical power in randomized control trials designed primarily to study $\mathrm{CV}$ and composite outcomes, residual bias in observational studies primarily designed to study cancer outcomes and differences between the characteristics of patients enrolled or included in the studies.

Cancer was assessed as a safety outcome in Liao et al's meta-analysis in which a directional protective effect was observed (four trials; RR 0.91 , CI 0.77 to 1.08 ). ${ }^{8}$ Suggested mechanisms for CV and non-CV benefits of pioglitazone, based on preclinical and clinical studies, include improvements in insulin resistance, decreased hyperglycemia, fat redistribution, renoprotection and improved liver function. ${ }^{9}$ A protective effect of pioglitazone for non-CV mortality is therefore plausible. Further research is required in this area before conclusions can be drawn.

\section{Conclusions}

This extended analysis following a large, observational multidatabase European cohort study found that prescribing pioglitazone compared with an alternative treatment decision at a similar stage of disease progression was associated with a reduction in both $\mathrm{CV}$ and non-CV mortality. Further observational and prospective studies that are specifically designed to test the association between pioglitazone use and patient-focused outcomes such as cause-specific mortality are suggested. Observational study designs should consider the nature of prescribing over time in the countries of interest and effect modification by prior TZD prescribing, history of diabetic complications and history of macrovascular disease.

Acknowledgements Dr Tim Williams (CPRD), Susan Eaton (CPRD), Leanne KoolHouweling (PHARMO Institute), Irene Bezemer (PHARMO) and Paul Dolin (Takeda) were involved in the original pan-European study. The Swedish National Diabetes Register provided detailed data for this analysis. The authors thank all patients and participating staff who have contributed to the register.

Contributors All authors planned and designed this post-hoc analysis. SC, MM, $\mathrm{ML}, \mathrm{RW}$ and $\mathrm{HS}$ performed the data management and data analysis, and all authors interpreted the data. All authors drafted the manuscript, revised the paper critically for important intellectual content and approved the final version of the manuscript. PK supervised the analysis and is the guarantor.

Funding Takeda Development Centre Europe Ltd.

Competing interests PK, FH, SC and MM are employed by EPID Research, EMH is employed by PHARMO Institute, RW and HS are employed by CPRD, and ML and SB are employed by Karolinska Institute. EPID Research, PHARMO Institute, CPRD and the Centre for Pharmacoepidemiology (CPE) at Karolinska Institute perform commissioned pharmacoepidemiological studies and thus their employees have been and currently are working in collaboration with several pharmaceutical companies (including Takeda). DB is employed by Takeda Pharmaceutical Company Limited.

Ethics approval In Finland, the study protocol was approved by the Ethical Review Board of the Hjelt Institute, University of Helsinki Medical Faculty (Dnro 96/13/00/2013). The research permission numbers to use the data were obtained from Statistics Finland (Dnro TK/53-373-13), National Institute for Health and Welfare (Dnro THL/634/5.05.00/2016), Social Insurance Institute (Dnro Kela 25/522/2013), Population Register Centre (Dnro 726/410/13). In Sweden, the study was approved by the Regional Ethical Review Board at Karolinska Institute in Stockholm, Sweden. DNR: 2011/82-31/3, 2011/752-323 and 2013/347-32. In the UK, the study protocol was reviewed and approved by the Independent Scientific Advisory Committee (ISAC) which considers and provides advice to the Medicines and Healthcare products Regulatory Agency on research projects which propose the use of data obtained from the CPRD (ISAC protocol numbers 13_044 and 14_065). In the Netherlands, the PHARMO compliance committee approved use of the PHARMO Database Network for the study and confirmed no ethics approval was needed.

Provenance and peer review Not commissioned; externally peer reviewed. Data sharing statement Criteria and process for sharing the analytical country specific datasets and meta-analysis dataset for third parties are defined in the study protocol available at the ENCePP E-Register of Studies.

Open Access This is an Open Access article distributed in accordance with the Creative Commons Attribution Non Commercial (CC BY-NC 4.0) license, which permits others to distribute, remix, adapt, build upon this work non-commercially, and license their derivative works on different terms, provided the original work is properly cited and the use is non-commercial. See: http://creativecommons.org/ licenses/by-nc/4.0/

(C) Article author(s) (or their employer(s) unless otherwise stated in the text of the article) 2018. All rights reserved. No commercial use is permitted unless otherwise expressly granted.

\section{REFERENCES}

1. Leal J, Gray AM, Clarke PM. Development of life-expectancy tables for people with type 2 diabetes. Eur Heart J 2009;30:834-9.

2. Panzram G. Mortality and survival in type 2 (non-insulin-dependent) diabetes mellitus. Diabetologia 1987;30:123-31.

3. Roper NA, Bilous RW, Kelly WF, et al. Excess mortality in a population with diabetes and the impact of material deprivation: longitudinal, population based study. BMJ 2001;322:1389-93.

4. Korhonen P, Heintjes EM, Williams R, et al. Pioglitazone use and risk of bladder cancer in patients with type 2 diabetes: retrospective cohort study using datasets from four European countries. BMJ 2016;354:i3903.

5. Strongman $\mathrm{H}$, Korhonen $\mathrm{P}$, Williams $\mathrm{R}$, et al. Pioglitazone and risk of mortality in patients with type 2 diabetes: results from a European multidatabase cohort study. BMJ Open Diabetes Res Care 2017;5:e000364.

6. Yang J, Vallarino C, Bron M, et al. A comparison of all-cause mortality with pioglitazone and insulin in type 2 diabetes: an expanded analysis from a retrospective cohort study. Curr Med Res Opin 2014;30:2223-31.

7. Hippisley-Cox J, Coupland C. Diabetes treatments and risk of heart failure, cardiovascular disease, and all cause mortality: cohort study in primary care. BMJ 2016;354:i3477.

8. Liao HW, Saver JL, Wu YL, et al. Pioglitazone and cardiovascular outcomes in patients with insulin resistance, pre-diabetes and type 2 diabetes: a systematic review and meta-analysis. BMJ Open 2017;7:e013927.

9. Rizos CV, Kei A, Elisaf MS. The current role of thiazolidinediones in diabetes management. Arch Toxicol 2016;90:1861-81.

10. Petri $\mathrm{H}$, Urquhart J. Channeling bias in the interpretation of drug effects. Stat Med 1991;10:577-81.

11. Brown AF, Ettner SL, Piette J, et al. Socioeconomic position and health among persons with diabetes mellitus: a conceptual framework and review of the literature. Epidemiol Rev 2004;26:63-77.

12. World Health Organisation. International statistical classification of diseases and related health problems - 10th revision. 5th edn, 2016. http://apps.who.int/classifications/icd10/browse/Content/statichtml/ ICD10Volume2 en_2016.pdf?ua=1\&ua=1 (cited 13 Nov 2017).

13. Brooke HL, Talbäck M, Hörnblad J, et al. The Swedish cause of death register. Eur J Epidemiol 2017;32:765-73.

14. Office for National Statistics. Mortality statitistics in England and Wales QMI [Internet], 2017. https://www.ons.gov.uk/peoplepopulatio nandcommunity/birthsdeathsandmarriages/deaths/qmis/ mortalitystatisticsinenglandandwalesqmi (cited 2017 Nov 13).

15. Official Statistics of Finland (OSF). Causes of death. ISSN=17995078. http://www.stat.fi/til/ksyyt/laa en.html

16. Dormandy JA, Charbonnel B, Eckland DJ, et al. Secondary prevention of macrovascular events in patients with type 2 diabetes in the PROactive Study (PROspective pioglitAzone Clinical Trial In macroVascular Events): a randomised controlled trial. Lancet 2005;366:1279-89.

17. Erdmann E, Harding S, Lam H, et al. Ten-year observational followup of PROactive: a randomized cardiovascular outcomes trial evaluating pioglitazone in type 2 diabetes. Diabetes Obes Metab 2016;18:266-73. 